\title{
Supplementary Information for \\ Grounding-line flux formula applied as a flux condition in numerical simulations fails for buttressed Antarctic ice streams
}

Ronja Reese ${ }^{1,2}$, Ricarda Winkelmann ${ }^{1,2}$, and G. Hilmar Gudmundsson ${ }^{3}$

${ }^{1}$ Potsdam Institute for Climate Impact Research (PIK), Member of the Leibniz Association, P.O. Box 6012 03, D-14412 Potsdam, Germany

${ }^{2}$ University of Potsdam, Institute of Physics and Astronomy, Karl-Liebknecht-Str. 24-25, 14476 Potsdam, Germany

${ }^{3}$ Extreme Environments, Northumbria University, Newcastle, UK

Correspondence to: Ricarda Winkelmann (ricarda.winkelmann@pik-potsdam.de) 

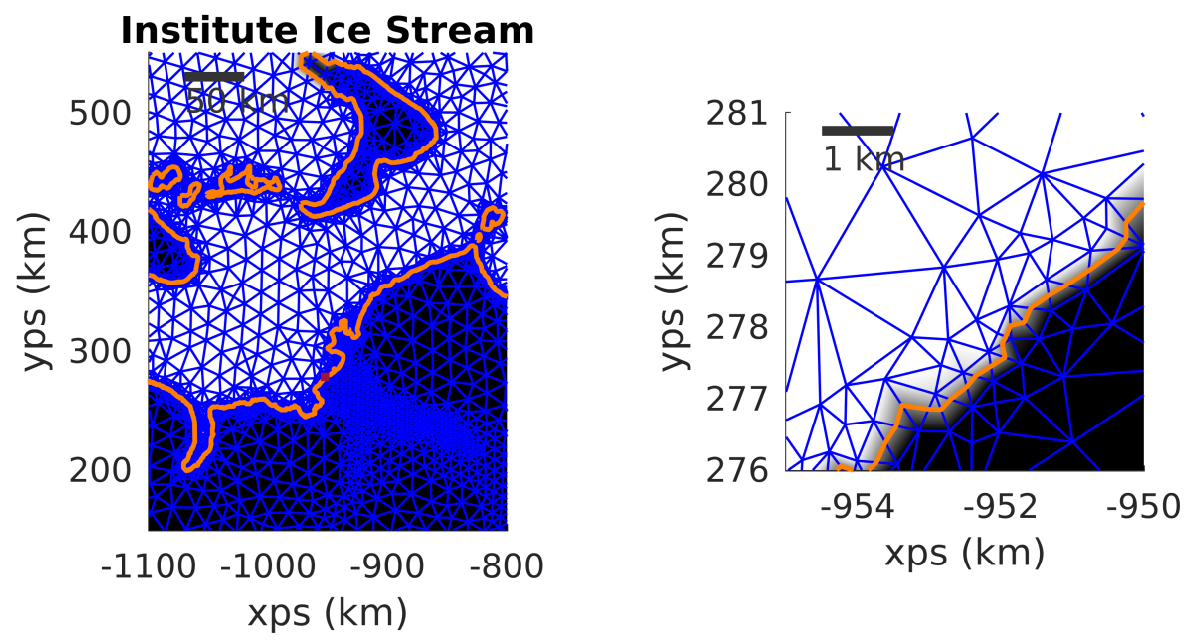

Figure S.1. Mesh elements around Institute Ice Stream are shown in blue. The grounded regions are indicated in black and the floating nodes in white. The grounding line is shown in orange. The right panel shows a zoom into the region indicated in red in the left panel. 


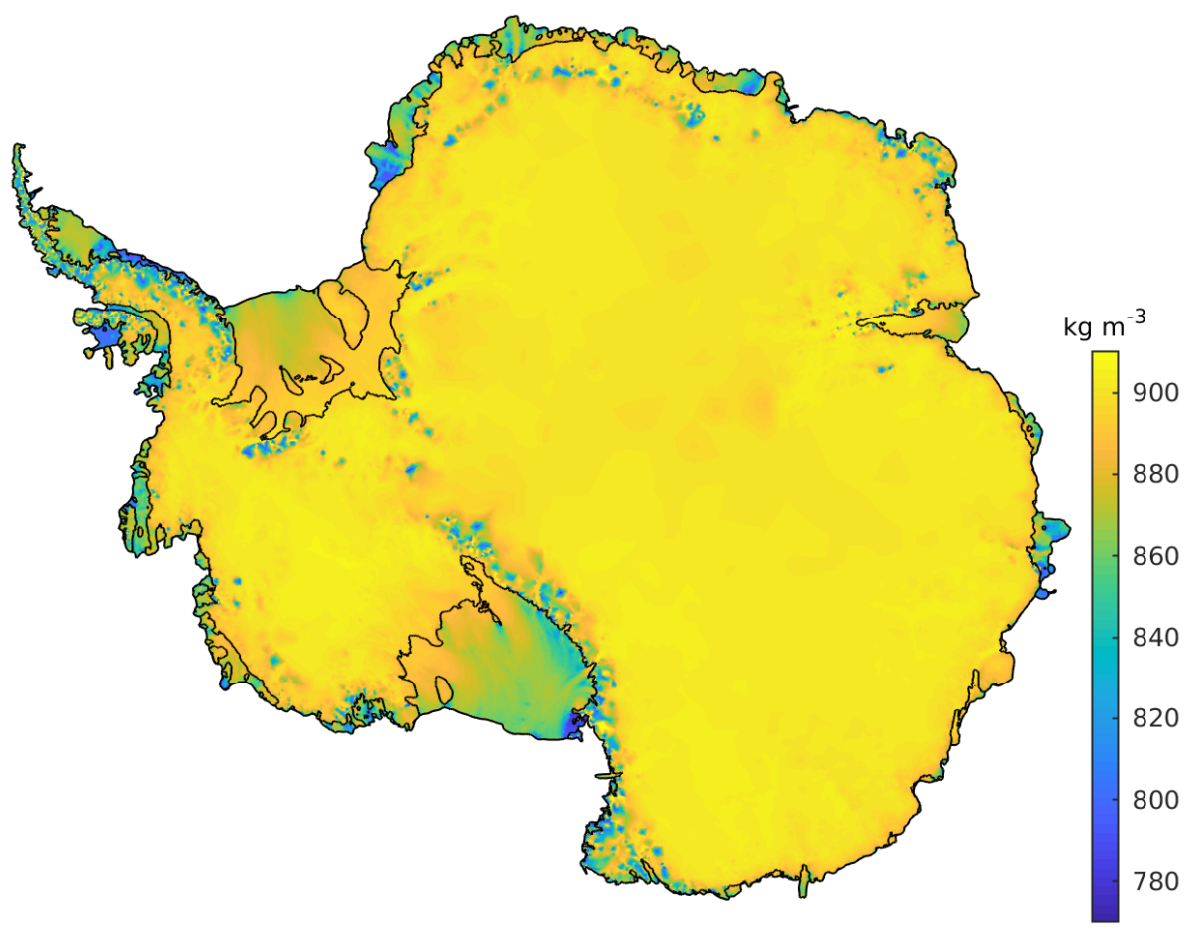

Figure S.2. The vertically averaged densities were calculated from RACMO2 firn thickness fields (Lenaerts et al., 2012) assuming an ice density of $910 \mathrm{~kg} \mathrm{~m}^{-3}$ and a firn density of $500 \mathrm{~kg} \mathrm{~m}^{-3}$. 


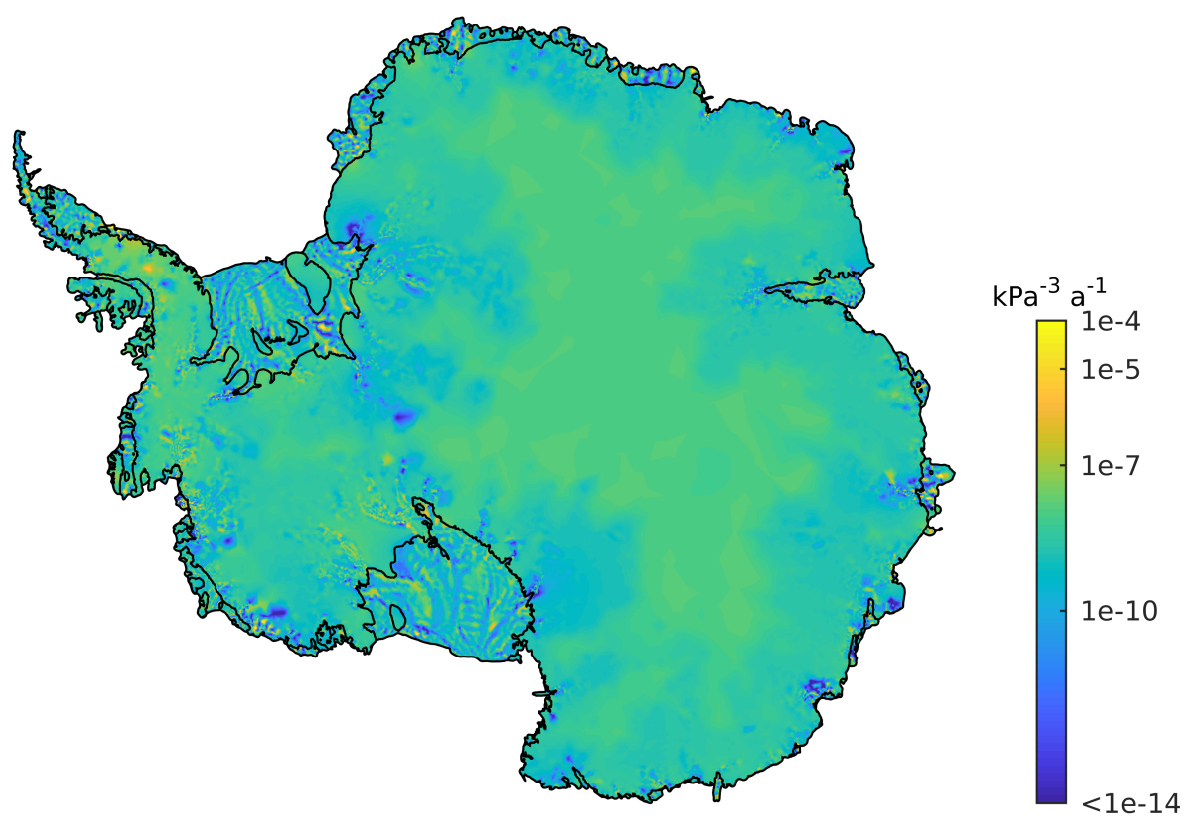

Figure S.3. The ice rate factor $A$ in Glen's flow law is estimated by simultaneous inversion together with $C$, assuming the exponent in Glen's flow law to be $n=3$. Similar to the basal slipperiness, the rate factor $A$ is a nodal-based quantity. The patches in the continent's interior are values of single nodes. 


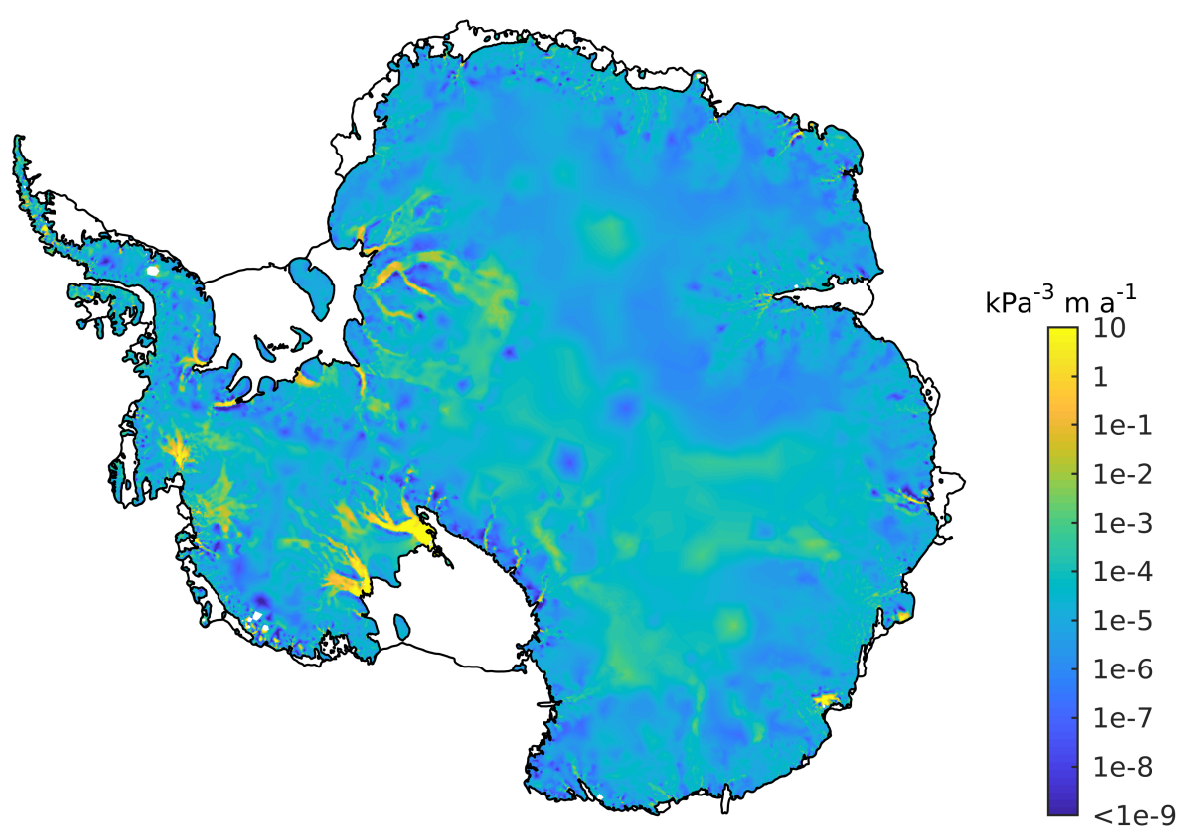

Figure S.4. The basal slipperiness distribution is obtained by inversion with the ice-flow model Úa assuming a Weertman-type sliding law with exponent $m=3$. The slipperiness is nodal based-value; the patches visible in the interior are values of single nodes. 


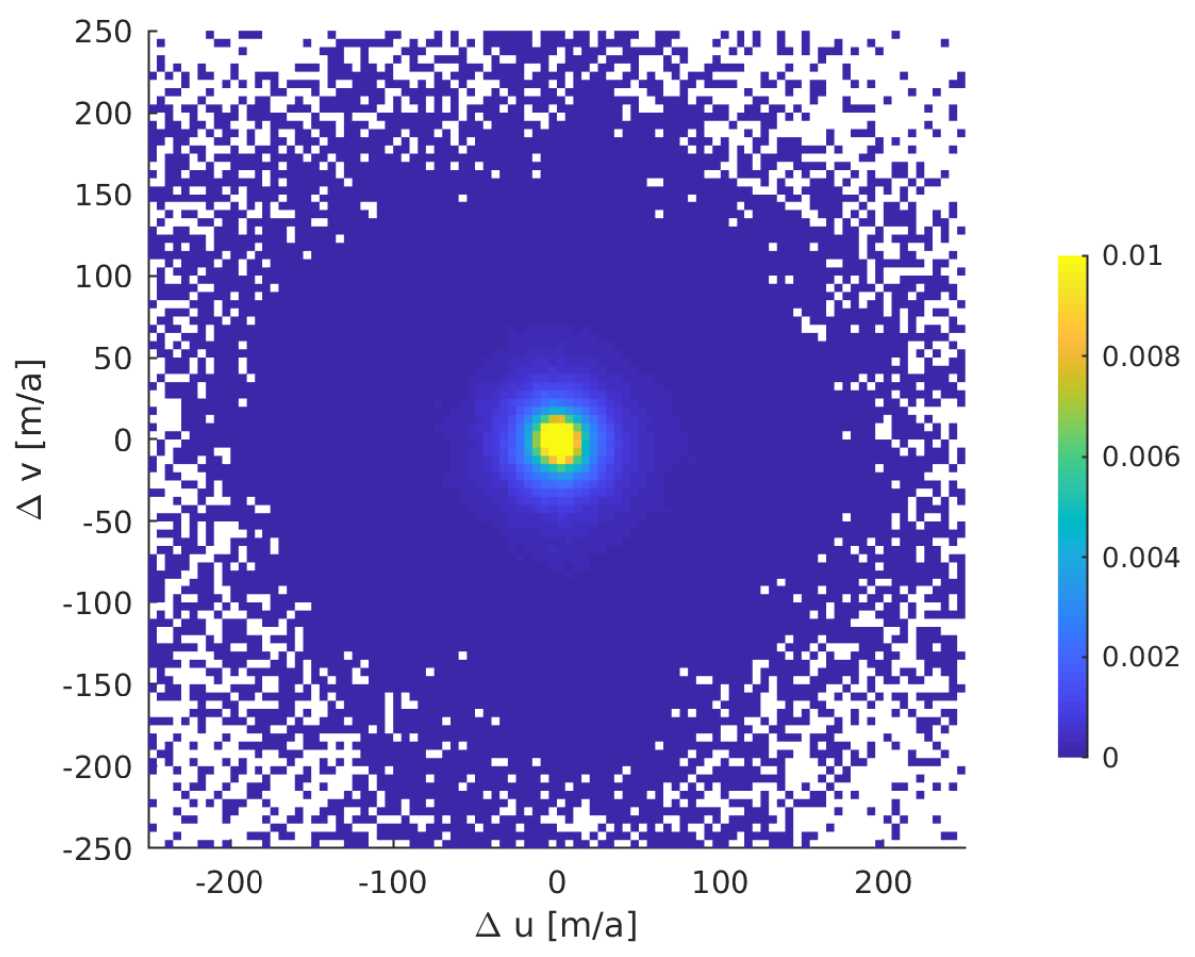

Figure S.5. Bivariate, normalized histogram of velocity residuals for all nodes of the Antarctic-wide mesh. 